\title{
Pilot study on an online transition course in mathematics
}

\author{
$\underline{\text { Stefan Berres }}^{\text {a }}$, Sophie Rehberg ${ }^{\text {a }}$ and Elias Colipe ${ }^{\text {a }}$ \\ ${ }^{a}$ Universidad Católica de Temuco, Chile \\ Email: sberres@uct.cl
}

\begin{abstract}
This report documents the experience generated by a pilot study of an online mathematics course offered to last and second last year high school students. The course counted with 109 participants from Chile, Colombia and Ecuador. The goal of the course is to support students in secondary education in learning mathematics with the help of a virtual platform, enabling them to continue with higher studies. As a main activity of the pilot study, the project team in Chile realized the online course during seven workshops, where the students working in a computer laboratory were observed and supervised.
\end{abstract}

A main conclusion is that the generation of working knowledge on how to manage the learning process using an online platform is a learning issue by its own, with implications that should not be underestimated. Even though most students are familiar with social network applications, there is no ability transfer when it comes to deal with productivity tools. One reason is the dependence of abilities on the used device. Dealing with smartphones is different to using desktop computers. For example, some workshop participants did not manage to scroll down the screen in order to find an exit button to confirm that a task has been completed and can be send to evaluation. Another example is that some do not manage the login procedure for the simple reason that they did not remember the password. They are not used to deal with passwords since usually smartphone applications do not request them. The implication for the overall project was that the session supervisors needed to track technical details including the login procedure.

One interesting observation made was that most of the course used to quit their work immediately after the break time bell. This behavior falsifies the supposition that individual and self-organized work might overcome the usual classroom conditioning that aims to minimize the time spend in classroom.

The adaption to a more autonomous working style, where the students work self-guided within the virtual environment took in average two sessions. Towards the end of the course, the students turned out to be motivated and in their majority interested in online learning, recognizing the complementary support to traditional classroom teaching.

Keywords: Distance education, STEM, transition course, math education 


\section{INTRODUCTION}

Recently, at the UN Sustainable Development Summit from September 25-27 2015, held at the UN headquarters in New York, out of the 17 Sustainable Development Goals, goal 4 promotes to 'Ensure inclusive and equitable quality education and promote lifelong learning opportunities for all'. One sub-goal particularly addresses the access to tertiary education, stating the following: 'By 2030, ensure equal access for all women and men to affordable and quality technical, vocational and tertiary education, including university.'

The advancement of communication technologies generates new opportunities in the development of society, like the gradual overcoming of inequalities in education. These inequalities typically persist when students come from vulnerable sectors because they are negatively affected by the lack of quality education, which in turn is a product of various factors, like teacher education focusing on pedagogical rather than disciplinary skills, low expectations of parents, socioeconomic income, among others (Muñoz and Redondo 2013).

Open Educational Resources (OER) might serve as a motivating support for students in their learning process and thus allow the improvement of the teaching-learning process (Friesen 2009; Rolfe 2012). A current trend is to integrate learning activities into social networks (Arnold and Paulus 2010).

Technology leverages the appropriation process and is permeating all social groups and institutions with a positive impact. The goal of the transition course is to support high school students in their process to the access higher education through the provision of mathematical learning, following a problem solving methodology and using a simulator of entrance exams. This report documents the experiences obtained during the design, implementation and execution of a pilot study of a transition course.

\section{METHODS}

\subsection{Course design}

The transition course is aimed at students of secondary education in order to facilitate their transition towards higher education. When entering the platform, the user finds an introduction, which explains how the courses and tools are working. There is a dynamic part and a static part of the course. In the static part the student finds the contents arranged in a tree structure that organizes the topics and subtopics, which have links to learning resources that facilitate the learning of the corresponding content. After the student has studied and hopefully understood the content, he or she can move to a test simulator, which provides tests from a question bank. In the case of insufficient understanding of a subject or having some doubts about one of the test questions, she or he can, besides revisiting the proposed resources, ask questions in the forum and discuss concerns with peers through the forum or an equivalent tool.

In the dynamic part of the course design, several courses on different thematic approaches, like 'Geometry', 'Statistics and Probability', 'Algebra and Functions' are offered, which are supposed to be repeated several times a year. The student can take the courses successively in any order or in parallel, according to individual abilities and needs.

In each subtopic, a warming up problem related to everyday life is presented. Since its solution is not immediate, the student is invited to deepen the topic and also to generate an interaction with peers by discussing the problem in a forum. This setting allows to apply the teaching methodology of Brousseau (2007) or the problem-solving method according to Pólya (1957), which proposes to split the problemsolving process into 4 phases, namely understanding the problem, creating a plan to solve it, carrying out the plan, and evaluating the solution. At the end of each topic, a summary of the content is provided in order to structure the related theory. The summary also helps students with irregular participation to retake and continue the course.

The pilot study dealt with geometry. The general topic was divided into five themes, namely angles and triangles, similarity, isometric and nomothetic transformations, space geometry, and plane trigonometry. The learning progress is controlled by comparing the results of an initial assessment with the results of a final evaluation. Both evaluations have the same level of difficulty.

The course units were designed having the following structure:

- Introduction to the subject, which motivates the need to work with the content. Description of the subject, emphasizing its potential applicability in everyday life.

- Initial assessment of previous knowledge required for the unit. The assessment includes a feedback with links to resources. 
- Initial problem to invite socialization of the subject. It counts with sufficient complexity to implement problem-solving strategies, e.g. according to Pólya.

- The contents are sequenced corresponding to the topic and the solution structure of the proposed problem. Links to learning resources are provided.

- Examples of exercises which are resolved step by step, eventually with help provided on different abstraction levels.

- Exercises in order to practice basic techniques; it is desirable that the platform provides a tool that visualizes the steps.

- Exercises with an increasing order of complexity that focus on both applications and on fundamental concepts in order to deepen the understanding. These exercises and questions can be discussed in the forums, or within a virtual classroom with virtual whiteboard.

- A well-crafted solution to the initial problem; there might be several versions, at the best the solutions are elaborated by the participants themselves.

- Final evaluation to assess the knowledge learned in the unit, complemented by additional challenging exercises and with feedback by links to resources.

- Summary on the treated topics and on the underlying theory.

\subsection{Platform selection}

For the selection of the platform, a set of key criteria has been identified, namely: (1) the platform allows to host content and to incorporate resources, (2) it facilitates learning and self-learning, (3) it provides a quick handling by users and group members, (4) eases the generation of standardized exams, (5) the platform code is available as open source.

In a test study, the learning platform ATutor was explored with respect to its capability of uploading contents and running exams; however, recurrent problems with the customization enforced to opt for the implementation of Moodle as learning platform on the project server in Temuco. As an alternative, the social networking software ELGG was also considered, but finally discarded because of its incapacity to transport learning contents adequately.

\section{OBSERVATIONS}

In the course, a total of 109 students of three countries have been enrolled, 42 from Colombia, 26 from Ecuador, and 41 from Chile. Whereas the course is designed and realized as online course, within this pilot study, a participant tracing was realized with a grade 11 course of the high school 'Liceo La Frontera de Temuco' during June and July 2013. During seven workshops realized in the schools computer laboratory, project members observed the interaction of the pupils with the online platform. In the following sections the observations realized during the accompanied sessions are documented.

\subsection{Handling of online platforms in general}

Not all students had an e-mail account and some of those who had one did not remember their password. Since their cell phones permits them to permanently save the password, they forget about it. This caused problems to register to the platform. Only at the end of the second workshop all students were registered. Moreover, during several sessions arose the problem to enter the platform due to forgotten passwords.

Although young people use electronic equipment as next generation smartphones, many students do not have much experience in managing information and communication technology, as soon as it comes to a usage in a labor environment with purposes on productivity. Deficiencies in careful reading and appropriate understanding introductory instructions and feedback messages made them dependent on the assistance by another person in order to assure progress. However, in the course of the workshop the students gradually got used to the platform and the presentation of its resources. Most of the students got used to the platform by the fourth workshop. By then, the students explored the material with greater ease and consulted the project team members only for very specific questions.

The lack of careful reading has the consequence that students do not show a self-learning ability. For example, they had to read and understand an introduction to the course methodology. There was no evidence whether the reading was done or not, but certainly it was questionable whether an adequate understanding was achieved. As indication, during the first workshops, while not adapted to the course methodology, frequently a personal step-to-step guidance was required, asking after each minor step what to do next. 


\subsection{Habits during the workshop}

Careful reading turned out to be a little developed skill. This makes the handling of the platform difficult, because messages have to be read in order to continue or terminate an activity by pressing a button labeled 'Send all and finish'.

The students preferred contents presented as video, but a low bandwidth did not always allow watching them completely. The contents presented in form of written documents were less popular. In spite of bandwidth problems they tried to watch the videos instead of reading through other available resources. Following the suggested links leading to specific contents, they did not expect that a link might take them to a list of contents. Also the amount of presented information influenced the motivation negatively.

As an evidence of a lack of self-study skills, students did not like to revisit the available resources after completing an evaluation. Although when the performance in the evaluation was rather poor, they preferred to move on in the course. In order to overcome the lacking self-learning ability, for the more delayed students a detailed guideline was provided specifying the steps to follow. During the course, most of the students got appropriated to the platform and the course, moving towards a more autonomous work.

\subsection{Motivation}

The students showed confidence from the first day of interacting with project members in various levels, by greeting, asking and talking to them. In the first workshop the students expressed their interest in participating in the course. They registered or at least attempted to register. Some had to create email accounts or recover passwords for their existing but rarely used accounts. Overcoming technical obstacles they began with the survey and the initial evaluation for the first unit.

The initial motivation dropped when they realized that they had to study and learn unknown math contents by activities that require certain attention and concentration. But as they got used to the platform and the methodology of the course the initial motivation was gradually recovered. The students helped each other both at technical problems and at questions on the mathematical content.

The affinity to videos for content exposition made the students to use their hearing aids of their cell phones. While waiting for the course platform to load, several students got bored and began to distract themselves by other activities such as getting to other sites, listen to music, looking up recipes, watching non-educational videos, which in turn further decreased the speed of the internet. They eventually closed those additional windows once surprised.

Usually, the whole course quitted their work immediately after the break-time bell. This behavior falsifies the supposition that individual and self-organized work might overcome the usual classroom conditioning that aims to minimize the time spend in classroom. As a pattern, some even stopped 10 to 15 minutes before the end of the session with the argument that they would not complete a task anyway as a consequence of the low speed internet in some computers, which negatively influenced the motivation. However, occasionally students were not concerned about going out to recess until they completed a pending task.

\section{RESULTS OF FINAL EVALUATION}

In the last workshop session a survey with qualitative and quantitative questions was conducted, answered by 31 students. The first question of the survey was open, asking what the participants liked and what they did not like about the course. Most students mentioned positive aspects, few wrote what they did not like it. More than half of the participants mentioned learning new contents as a positive aspect, some mentioned that the treated content served as refresher because they either had it forgotten or never seen in class.

This is in concordance to the answer of another open question on the issues learned during the course, where all responses are related to mathematical content, whereas no student refers to the appropriation of the platform and technological aspects. The treatment of the content through an online platform is found more educational and entertaining; one participant recognized that he could concentrate more than in traditional classes and thus better understand the content. Another participant liked the large number of materials that could be occupied for learning and mentions the possibility of sharing information through the forum. Yet another one considered it valuable that the evaluations have an explanatory feedback.

A positive aspect mentioned apart is that the exercises are challenging their knowledge. The negative aspects were related to the level of complexity of the content; for $20 \%$ of the participants the questions were felt to be very complicated and not possible to understand. They also criticized that the teachers did not sufficiently clarify doubts and did not allow to watch a football game. The latter answer is congruent to an answer to the 
last question on other comments and suggestions, namely applying for the permission to listen to music while working.

The impression on the complexity level of the resources, exercises and problems was evaluated on a scale from 1 to 5 corresponding to very basic to very complex. More than the half of the participants assessed the complexity of the course to be difficult or very difficult. Almost nobody assessed the level to be below average. Regarding explicit comments, they include an appreciation that the exercises present very good challenges giving the opportunity to remember and connect the contents treated previously in classroom. Even though, by others, the exercises are assessed to be more or less difficult because some materials has not been passed in previous courses, or, eventually the material has been passed, but it is not remembered, some parts understood, others not. Other comments judged the exercises to be very entertaining and helped to learn much, expressed the recognition of the need to read the information first, or presented the concession of a general dislike of mathematics.

\subsection{Platform familiarity}

Asked about difficulties when starting to work on the platform, $62 \%$ of the students mainly attributed their difficulties to the mathematical content because they had to solve exercises and problems, and begin to understand contents, which before they were unaware or had forgotten. One student mentioned the distraction by other sites like social media. Another states that one difficulty was to see the videos without listening, it seems that he was the one who had no headphones. Around $30 \%$ mention as the main obstacle to enter the platform for the forgotten password, which is consistent with observations made by the project team.

Regarding the ownership of the platform, $87 \%$ recognized to have achieved an appropriation after the third workshop, see Table 1. This answer is consistent with the observations of project members during the course.

Table 1. Question: During which workshop did you comprehend how the platform works?

\begin{tabular}{|l|l|l|l|l|l|l|}
\hline Workshop Number & 1 & 2 & 3 & 4 & 5 & 6 \\
\hline Cumulative \% & 17 & 67 & 87 & 90 & 90 & 93 \\
\hline
\end{tabular}

More than three-quarters agree that they have learned something during the course, indicating that the experience was useful or at least fairly useful, whereas the other students are unsure of their progress and found the course little useful.

\subsection{Learning preferences}

The general learning preferences are quite diverse with only a slight bias towards the proposed strategy of autonomous online learning as an eventually desirable answer. In fact, $23 \%$ of the participants prefer to learn in a traditional way without online component because in normal classes they can interact directly with the teacher and with peers. One participant confirmed that he prefers to learn in a traditional way because he is used to it from primary school. Another states explicitly that, when he does not know something then he prefers to seek help from a teacher. 33\% prefer to learn on their own in the online course because of the adaptable speed of learning through internet, where it is convenient to continue reading and occasionally to reread if something is not understood. $27 \%$ prefer to learn in campus classes and incorporating online learning resources because the incorporation of online elements is entertaining, but they prefer keeping the option to clarify doubts with a teacher. $17 \%$ of the students prefer to learn mainly the traditional way, complementing their learning autonomously with an online course, i.e. they like the idea that the learning could be complemented by an online course. The expressed views are very balanced. Even though there is no clear trend, one can say that about three quarters would like to incorporate virtual elements in their learning in some form.

There are divided believes on whether a student can learn a mathematical content by only using an online platform. More than half (52\%) of the participants believe that one can learn math using an online platform, because there is the possibility to view the same content repeatedly until it is understood. There is the possibility to advance better in the appropriation of content considering its educational and entertaining presentation. One student remarks that for him it is better to learn on his own, when he is alone in the room, in comparison to a classroom situation, which is usually relatively loud such that one gets unfocused; for his part he learned a lot since in the classroom he would not pay as much attention. Still, $20 \%$ consider the platform and the course as an additional support to traditional classes rather than being the only source. Finally, 28\% is not at all convinced in online learning, because they need the support of a teacher or another 
person to interact with. One participant raises the question of connectivity options, which is valid for many other participants, observing that some students at home do not have the means to be connected to internet because some live in remote places and others do not have computers.

On the question whether virtual resources help to understand any topic of interest, $57 \%$ gave an unconditional approval, $37 \%$ conditioned the potential benefit to the type of the resource. Asked explicitly, $93 \%$ would use the platform to support the learning of a topic treated in the course. In the survey, this question was repeated in several variants in order to allow a cross-validation: Asked whether they would use this or another platform to obtain help in math, $90 \%$ of students were confirmative. In yet another question variant, $72 \%$ responded that they would autonomously participate in another course like this.

This answer pattern can be explained by the progress, which was made with the course: $63 \%$ of the students feel to have learned something in the course, no student feels to have learned nothing, see Table 2 . The $30 \%$ who scored relatively undecided might not feel quite sure about their learning or simply marked in the middle of the answer scale because they are indecisive.

Table 2. Question: Did the resources available on the platform help you to understand the contents and correct your mistakes?

\begin{tabular}{|l|l|l|l|l|l|l|}
\hline No, nothing & 1 & 2 & 3 & 4 & 5 & Yes, very much \\
\hline & $0 \%$ & $7 \%$ & $30 \%$ & $43 \%$ & $20 \%$ & \\
\hline
\end{tabular}

\section{COMPLEMENTARY OBSERVATIONS}

Besides the detailed observation and feedback that was obtained in the reported case study, several other project partners simultaneously rolled out the same transition course. In particular, it was offered in three other cities, namely Bogotá, Popayán and Quito. Different course materials worked out by various project partners have been tested at different locations. The transition course was announced by a series of school visits, where typically the project was presented and some information on the transition course given. Whereas the news on the soon starting transition course was well received, the effective participation was far less abundant than expected.

The information on the effective participation is rather scarce since there was no active tracing of the activities. One main figure is that, in the context of the launch of the transition course, around 300 persons approached by the Popayán team registered in the platform, but of them only 38 were inscribed in the course. Out of these only one person completed all the evaluations, but he was evidently not part of the target group, as his IP address corresponded to a higher education institution.

Many received comments from the project partners referred to the course structure and material, particularly to early stage activities regarding the course design, but not to on-site observations on the student performance. Thus, there is no report on login difficulties, which might have confirmed the on-site observations presented in the case study, neither there is any complementary documentation on the process of getting acquainted with the platform. This might be explained by the hypothesis that there simply were no difficulties to report. However, it is more probable that students unfamiliar with online platforms simply have not been reached, and that no particular pilot scale tracing has been done.

\section{REVISION OF PLATFORM AND COURSE DESIGN}

During the course preparation, an experimental study of ATutor as potential platform for hosting the course consumed a significant amount of time. Even a trial version of the transition course was mounted in ATutor. The reason for this consumed time is that, in an early stage of the project, Moodle was avoided to be a component of the main platform, representing a supposedly outdated pedagogy and providing an unfriendly design with lacking tools. It was judged that the platform Moodle changes the pedagogical focus from conductivism to constructivism, but not yet reaching 'connectivism', a learning setup, where the students are supposed to self-organize their own learning within self-constructed learning networks. Given the unexpected difficulties shown by ATutor, the decision to finally use Moodle as platform for the online course was fairly close to the scheduled start of the transition course. As a consequence, the material of some course units was not yet completed at the official start of the course, a delay, which also impacted the early announcements.

The platform Moodle counts with a broad set of features of a learning management system, being by now one of the most spread systems. Still, the single tools are not the most fluent in usability, in particular it does not have a user-friendly forum, a fact, which negatively affects the interaction between participants and the 
potential to create a virtual community. As alternative platform, ELGG was tested in other lines of the umbrella project as a tool, inviting the participants to create groups and interact within these groups; however it proved only partly to be able to emulate a social media network and thus fostering a virtual community. It did not compensate its lack of features of a learning management system.

Several aspects in the course design have been identified to be crucial for a smoother learning process, leading to the following suggestions:

- The platform, the methodology and the structure of the course should be adequately introduced.

- The structure of the units and the tools should be standardized.

- The questions should address the pedagogical situation adequately, the given information should be complete and avoid ambiguities.

- Links should not open in a new window to avoid confusion in navigating pages.

- The presentation of the resources should avoid distracting elements. A counterexample is when links are to sites where the relevant definition is barely visible or below distracting elements, giving the sensation that the indicated site is wrong.

- The nomenclature of the contents should be standardized to avoid confusion. One should not name the same type of resource by different synonyms, or refer to resources corresponding to different topics by identical names. This avoids the situation that students enter the evaluation of another section, not realizing that there are different evaluations for different sections available at the same time.

\section{CONCLUSION AND DISCUSSION}

In a transition course, typically contradicting goals have to be met. One is to prepare students to perform well in formally required standardized entrance exams for the higher education system, and another is to prepare students in the mathematical content of the university itself. In the case of Chile the entrance exam to the university requires skills trained in the line of conductivism, such as the application of algorithms and solution recipes rather than logical reasoning and problem-solving strategies, whereas at university the focus tends to be rather on the development of cognitive skills.

The successful participation in an online course requires some familiarity with the used platform. This implies that for a more intensive participation both the teachers and the students need to get acquainted with the platform in more detail with the goal that they consider and integrate the course in their other activities. The ultimate goal of having a virtual interaction of the course participants via forums could not be reached within this transition course. The expectation of the creation of a vibrant virtual community within the transition course was overly optimistic, given the age and maturity of the participants. However, some valuable feedback on the potential of the interaction of students of the target group with online platforms could be obtained.

\section{ACKNOWLEDGMENTS}

This pilot study has been developed within the project 'Virtual Classroom of Mathematics and Mentoring', financed by the ALFA III programme of the European Union. Also, support by the 'Convenio de Desempeo para la Educación Superior Regional UCT1302' is acknowledged.

\section{REFERENCES}

Arnold, N. and Paulus, T. (2010). Using a social networking site for experiential learning: Appropriating, lurking, modeling and community building. Internet and Higher Education, 13 (4), 188-196.

Friesen, N. (2009). Open educational resources: New possibilities for change and sustainability. International Review of Research in Open and Distance Learning, 10 (5).

Guy Brousseau (2007). Iniciación al estudio de la teoría de las situaciones didácticas, Buenos Aires, Libros del Zorzal.

Muñoz, P. and Redondo, A. (2013). Desigualdad y logro académico en Chile, Revista CEPAL 109, 107-123.

Pólya, G. (1957). How to Solve It. Garden City, NY: Doubleday.

Rolfe, V. (2012). Open educational resources: Staff attitudes and awareness. Research in Learning Technology, 20 (1), art. no. 14395. 